\title{
Microstrip Triplexer using a common triple-mode resonator
}

\begin{tabular}{|r|l|}
\hline Journal: & Microwave and Optical Technology Letters \\
\hline Manuscript ID & MOP-17-1599 \\
\hline Diley - Manuscript type: & Research Article \\
\hline Complete List of Authors: & $\begin{array}{l}\text { El-Tokhy, Amira; University of Greenwich, Engineering science } \\
\text { Wu, Ruiheng; University of Greenwich, Engineering Science } \\
\text { Wang, Yi; University of Greenwich Faculty of Engineering and Science, } \\
\text { Engineering Science; University of Greenwich }\end{array}$ \\
\hline Keywords: & Triplexer, Stub-loaded resonator \\
\hline \multicolumn{2}{|c}{} \\
\hline
\end{tabular}

SCHOLARONE ${ }^{m}$

Manuscripts 


\author{
Microstrip Triplexer using a common triple-mode resonator \\ Amira El-Tokhy ${ }^{1 *}$, Ruiheng $\mathrm{Wu}^{1}$, Yi Wang ${ }^{1}$ \\ ${ }^{1}$ Department of Engineering Science, University of Greenwich, Chatham \\ Maritime, Kent, ME4 4TB, UK \\ *a.e.a.eltokhy@gre.ac.uk
}

\begin{abstract}
An all-resonator based triplexer is presented using a double-stub-loaded resonator (DSLR) that acts as a common resonator at the junction of the three channels. The open stub DSLR has been analysed using even and odd-mode method to reveal the relationship between the three resonant modes. The design offers flexibility of frequency selection. The DSLR resonator is coupled with three sets of hairpin resonators to form the triplexer at 1.8, 2.1, and $2.6 \mathrm{GHz}$ for mobile communication applications. The measurement results are in very good agreement with the simulations.
\end{abstract}

\section{Introduction}

As wireless communication technology advances, devices supporting multiple standards and multiple bands are increasingly desired in mobile communications and satellite systems. For instance, a triplexer may be required to connect the stack of filters for the DCS1800 band, UMTS2100 band and some LTE bands. Traditionally a multiplexer is formed of channel filters which are designed separately and then combined using a transmission-line based junction. Different types of junctions have been used over the years, such as Tjunctions, manifolds or star junctions [1]. Recently a new concept, the so-called multi-port filtering network (MPFN), has been developed [2]. The main difference between the conventional two-port filter and the MPFN is the junction resonators, which are responsible for directing the signal to multiple ports. It has been the focus of many researches, as it has showed advantages on circuit size reduction as well as the capability of incorporating new device configurations [3].

The junction resonator, or as also called the common resonator, has a major role to play in the MPFN design. It can be a single [4] or multi-mode [5] resonator. A single-mode resonator is relatively easy to implement but often put restrictions on the achievable overall bandwidth of the device. For diplexers or multiplexers with widely separate channels, it is desirable to use multi-mode resonators that are capable of producing multiple resonances. It is a particular advantage to have controllable resonance frequencies. One of the most used is the stub loaded resonator (SLR). It consists of a halfwavelength resonator and an open or a shorted stub. The stub provides an additional frequency band to achieve a dual-band resonator. This gives the advantage of controlling the frequencies flexibly by tuning the lengths of the stubs [6-8]. An open-stub-loaded eight-mode resonator was presented in [9], which used group of open stubs and one shorted stub to create eight modes. In [10] a triple mode SLR was used but only for a diplexer design. Asymmetric stub-loaded resonators have been used in [11] to design a wide stopband tri-band filter. The combined open and short stub loaded resonators had been presented in [10], [12-14] for a triple mode resonator in filters, diplexers and power dividers. The stubs were used to control the second and third resonant frequencies. The same approach was used recently where a triple-mode SLR was used to design a triplexer in [15].
This letter presents an all-resonator based triplexer that uses a folded double-stub-loaded-resonator (DSLR) as it replaces the junction and distributes the signal into three paths. Previous works used mixed open and short stubs to give good isolation, but limited the structural topology. Different from previous work, all stubs used in this paper are open for the ease of implementation in microstrip lines.

\section{Design of tri-mode DSLR}

The single open stub loaded resonator has been studied extensively [16] as a dual-mode resonator. The tri-mode resonator with three resonant frequencies is designed here based on the double SLR (DSLR).

To achieve triple modes, two open stubs form a crossshape stub loaded resonator as shown in Fig. 1(a). The half wavelength resonator $(2 \times \mathrm{L} 1)$ resonates at $1.8 \mathrm{GHz}$. The two stubs are located in the middle of the half wave resonator. The lower longer stub (L2) helps to realise the $2.1 \mathrm{GHz}$ resonance, whereas the shorter upper stub (L3) to realise the $2.6 \mathrm{GHz}$ resonance. $\theta_{1}, \theta_{2}$ and $\theta_{3}$ are the corresponding electrical lengths. Due to the symmetric structure of the resonator, odd and even mode analysis has been used as shown in Fig. 1.

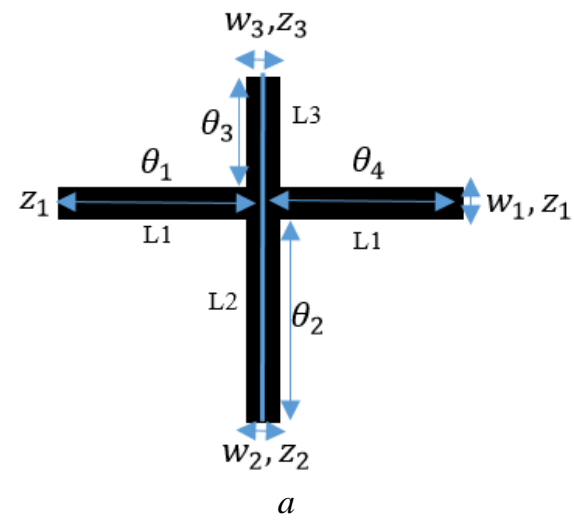



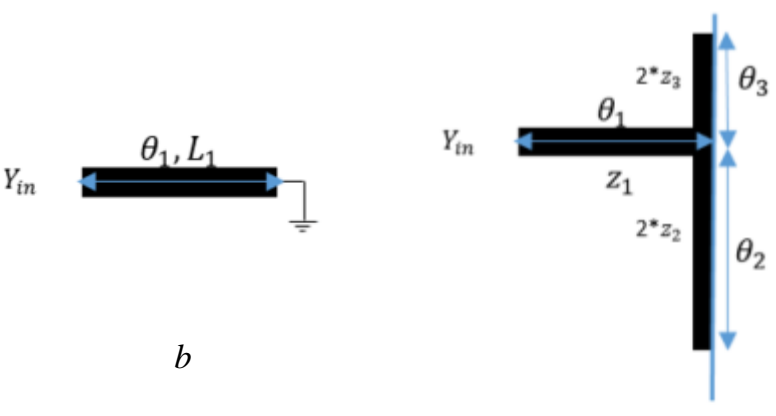

$c$

Fig. 1. Proposed triple-mode DSLR and its odd and even equivalent circuits.

(a) Layout, (b) Odd mode equivalent circuit layout, and (c) Even mode equivalent circuit layout

Fig. 1(b) shows the equivalent circuit for the odd mode, when the junction represents a short circuit. (1) can be used to obtain the odd mode frequency.

$$
f_{o d d}=\frac{(2 n-1) c}{2 L_{1} \sqrt{\varepsilon_{e}}}
$$

where $c$ is the speed of light and $\varepsilon_{\mathrm{e}}$ is the effective dielectric constant. The odd mode frequency is designed to be $1.8 \mathrm{GHz}$.

For the even mode, the equivalent circuit is reduced to a T-shaped single stub loaded resonator as shown in Fig. 1(c). Due to the open circuit boundary condition at the symmetric plane, the impedances of the stubs (with $\theta_{2}$ and $\theta_{3}$ ) are doubled to $2 Z_{2}$ and $2 Z_{3}$. There is no symmetry in this stub loaded resonator structure and its dual mode resonant property has been studied in [5]. The input admittance can be expressed as

$$
Y_{\text {in }}=-j Y_{1} \frac{Y_{1} \tan \left(R_{f} \theta_{1}\right)+Y_{2} \tan \left(R_{f} \theta_{2}\right)+Y_{3} \tan \left(R_{f} \theta_{3}\right)}{\tan \left(R_{f} \theta_{1}\right)\left[Y_{2} \tan \left(R_{f} \theta_{2}\right)+Y_{3} \tan \left(R_{f} \theta_{3}\right)\right]-Y_{1}}
$$

where $Y_{1}=1 / Z_{1}, Y_{2}=1 /\left(2 Z_{2}\right), Y_{3}=1 /\left(2 Z_{3}\right) . R_{f}$ is the ratio between the first two resonance frequencies of the single SLR. In this design, it is also the ratio between the second $(2.1 \mathrm{GHz})$ and the third $(2.6 \mathrm{GHz})$ resonance of the DSLR. Therefore, the resonant condition is:

$$
\frac{\tan _{f} \theta_{1}}{Z_{1}}+\frac{\tan _{f} \theta_{2}}{2 Z_{2}}+\frac{\tan _{f} \theta_{3}}{2 Z_{3}}=0
$$

This design assumes equal line width of the DSLR, i.e. $Z_{1}=$ $Z_{2}=Z_{3}$. The targeted two resonances are 2.1 and $2.6 \mathrm{GHz}$ in this work.

Fig. 2(a) illustrates the relationship between the resonance ratio $R_{f}$ and the electrical length of $\theta_{3}$ when the rest of the parameters are fixed. It should be noted that the three electrical lengths $\theta_{1}, \theta_{2}$ and $\theta_{3}$ are defined at the first resonance of the even-mode equivalent circuit, i.e. $2.1 \mathrm{GHz}$. Since $\theta_{1}$ is $90^{\circ}$ at $1.8 \mathrm{GHz}$, it is fixed at $105^{\circ}$ for $2.1 \mathrm{GHz}$. Also, $\theta_{2}$ is varied at $80^{\circ}, 90^{\circ}, 100^{\circ}$ and $110^{\circ}$ at $2.1 \mathrm{GHz}$. The ratio $R_{f}$ varies against $\theta_{3}$, illustrating the degree of freedom that the design has to resonate at controllable frequencies as shown in Table 1. From Fig. 2(a), when the desired $R_{f}=2.6 / 2.1=1.23, \theta_{3}$ is found to be $64.2^{\circ}$ at $2.1 \mathrm{GHz}$.
The calculated L1, L2 and L3 from (3) are $105^{\circ}(25.03 \mathrm{~mm})$, $90^{\circ}(21.44 \mathrm{~mm})$, and $64.2^{\circ}(15.28 \mathrm{~mm})$ at $2.1 \mathrm{GHz}$. Table 2 shows the solutions for $\theta_{3}$ as $\theta_{2}$ changes for $R_{f}=1.23$.

Table 1 The physical lengths at the three bands and its corresponding lengths.

\begin{tabular}{|c|c|c|c|c|c|}
\hline At 1.8 & $\begin{array}{c}\text { At 2.1 } \\
\mathrm{GHz}\left({ }^{\circ}\right) \\
\left({ }^{\circ}\right)\end{array}$ & $\begin{array}{c}\text { At 2.6 } \\
\mathrm{GHz} \\
\left({ }^{\circ}\right)\end{array}$ & $\begin{array}{c}\text { Calculated } \\
\text { lengths } \\
(\mathrm{mm})\end{array}$ & $\begin{array}{c}\text { Optimized } \\
\text { lengths } \\
(\mathrm{mm})\end{array}$ \\
\hline $\boldsymbol{\theta}_{1}$ & 90 & 105 & 103.3 & 25.03 & 25.0 \\
\hline $\boldsymbol{\theta}_{2}$ & 77.1 & 90 & 111.6 & 21.44 & 21.1 \\
\hline $\boldsymbol{\theta}_{3}$ & 55.0 & 64.2 & 79.6 & 15.28 & 15.3 \\
\hline
\end{tabular}

Table 2 Solutions for $\theta_{2}, \theta_{3}$ and the corresponding L2 and L3 for $R_{f}=1.23$.

\begin{tabular}{|c|c|c|c|}
\hline$\theta_{2}\left({ }^{\circ}\right)$ & $\theta_{3}\left(^{\circ}\right)$ & $\mathrm{L} 2(\mathrm{~mm})$ & $\mathrm{L} 3(\mathrm{~mm})$ \\
\hline 80 & 68.1 & 19.06 & 16.23 \\
\hline 90 & 64.2 & 21.44 & 15.28 \\
\hline 100 & 61.8 & 23.82 & 14.71 \\
\hline 110 & 60.0 & 26.20 & 14.29 \\
\hline
\end{tabular}

Based on the $R_{f} \sim \theta$ relationship, a DSLR resonator is designed using Sonnet. The S-parameter responses show the three modes in Fig. 2(b). Roger 3004C substrate with $\varepsilon_{r}=$ 3.55 and a thickness of $1.524 \mathrm{~mm}$ is chosen. The DSLR achieved the targeted frequencies with $\mathrm{L} 1=25 \mathrm{~mm}, \mathrm{~L} 2=21.1$ $\mathrm{mm}$, and L3 $=15.3 \mathrm{~mm}$ after optimization. These values are in good agreement with the calculations as shown in Table 1.
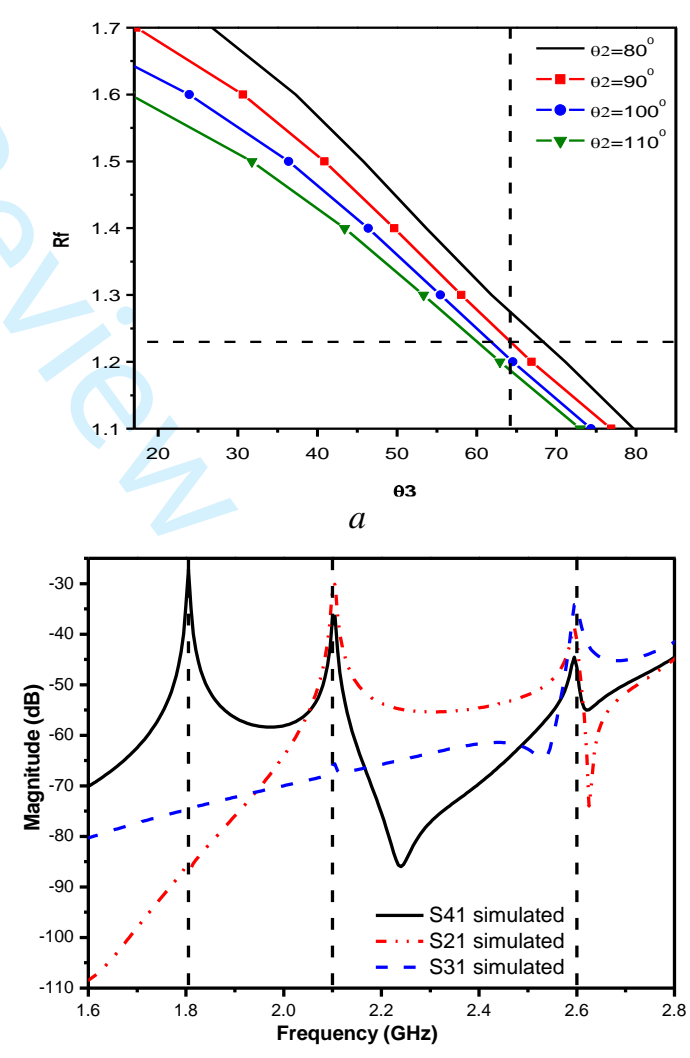

$b$

Fig. 2. The triple-mode DSLR physical lengths analysis and its simulated resonances.

(a) $R_{f}$ as a function of the electrical length $\theta_{2}$ and $\theta_{3}$. (b) The simulated resonances of the DSLR. 
Fig. 3 shows the simulated current distributions of the DSLR showing the current concentration in the lower stub at $2.1 \mathrm{GHz}$ and in the upper stub at $2.6 \mathrm{GHz}$. At $1.8 \mathrm{GHz}$, no current flows into the two stubs.
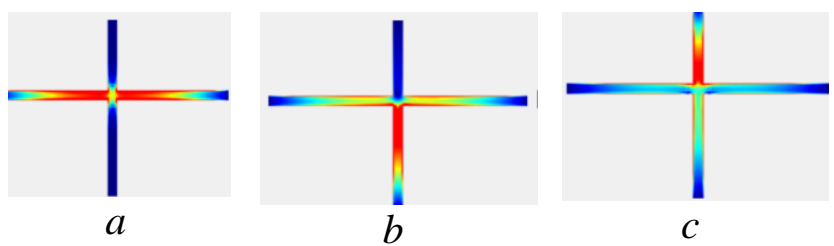

Fig. 3. Simulated current distribution of the DSLR.

(a) $1.8 \mathrm{GHz}$, (b) $2.1 \mathrm{GHz}$, and (c) $2.6 \mathrm{GHz}$

\section{Design of triplexer}

The triplexer is designed using an all-resonator based topology. A folded DSLR is used to replace the conventional impedance matching junction and distribute the signal into three paths. The first path is via the odd mode of the DSLR which is coupled with a half wavelength hairpin resonator. The second path is via the longer open stub coupled with a hairpin resonator at $2.1 \mathrm{GHz}$. The third path is via the shorter open stub of the DSLR. The topology is shown in Fig. 4. The white circles represent the resonators. The circle marked with XR represents the common triple-mode DSLR resonator while the circles with $\mathrm{R}$ are the single mode hairpin resonators.

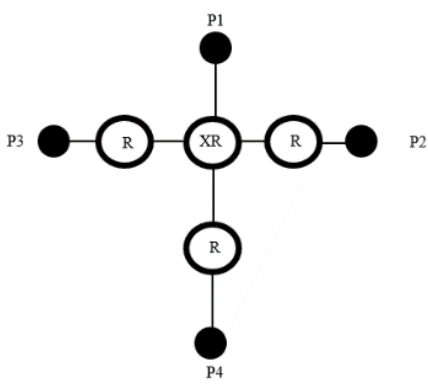

Fig.4. The proposed triplexer coupling topology.

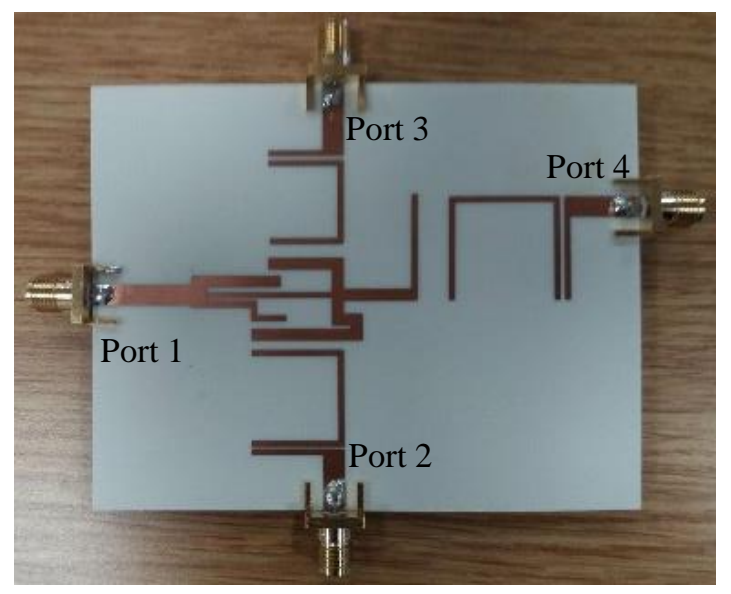

Fig.5. Layout of the proposed triplexer.

In this topology, the input port P1 is designed to couple to the three modes of the DSLR respectively. So, the coupling structure can be characterised using three independent $2^{\text {nd }}$ order filters all with a return loss of $20 \mathrm{~dB}$. So corresponding coupling matrix and external quality factor calculated as follows:

$$
\begin{aligned}
& \mathrm{M}^{1}=\left[\begin{array}{ccc}
0 & 0.023 & 0 \\
0.023 & 0 & 0.023 \\
0 & 0.023 & 0
\end{array}\right] \\
& \mathrm{M}^{2}=\left[\begin{array}{ccc}
0 & 0.0316 & 0 \\
0.0316 & 0 & 0.0316 \\
0 & 0.0316 & 0
\end{array}\right] \\
& \mathrm{M}^{3}=\left[\begin{array}{ccc}
0 & 0.0316 & 0 \\
0 & 0.0265 & 0 \\
0.0265 & 0 & 0.0265 \\
0 & 0.0265 & 0
\end{array}\right]
\end{aligned}
$$

and

$$
Q_{e x}=47.5,34.12,41.69
$$

After a process of $\mathrm{Q}$ and $\mathrm{M}$ extraction for each resonator individually; the final stage is to assemble all the resonators as illustrated in Fig. 5. A fork shape input feed has been used to reach the required external quality factor for each of the three modes. It should also be noted that nonuniform impedance lines have been used in the DSLR to facilitate coupling.

\section{Simulated and Measured Results}

Fig. 6 shows the simulated results using Sonnet in comparison with the measurements from a N5230A Network analyser. Very good agreement has been achieved. There is a slight shift in frequencies because of fabrication tolerance. The central frequencies are 1.88, 2.1, and 2.6 GHz; The FBW achieved are $0.86 \% .1 .4 \%, 0.96 \%$ respectively. The return losses are better than $15 \mathrm{~dB}$ for all three channels.

The $2.1 \mathrm{GHz}$ passband from Port 2 has an insertion loss of $1.3 \mathrm{~dB}$ and rejects the other two bands to below $-43 \mathrm{~dB}$. Likewise, the third band was extracted at Port 3 with $2.3 \mathrm{~dB}$ insertion loss while rejecting the other two bands to below $48 \mathrm{~dB}$. For the first band, the insertion loss is $3.2 \mathrm{~dB}$. The rejection is $21 \mathrm{~dB}$ to the $2.1 \mathrm{GHz}$ band and $36 \mathrm{~dB}$ to the 2.6 $\mathrm{GHz}$ band.

Regarding ports isolation, the isolation between Ports 3 and 4 is found to be over $43 \mathrm{~dB}$. This is over $42 \mathrm{~dB}$ between Port 2 and 3. The isolation between Port 4 and 2 is the worst at around $1.88 \mathrm{GHz}$, which is at $27 \mathrm{~dB}$. The isolation performance in Fig. 6 (c) shows that, although the design used all open circuits, reasonably good isolation is still achieved.

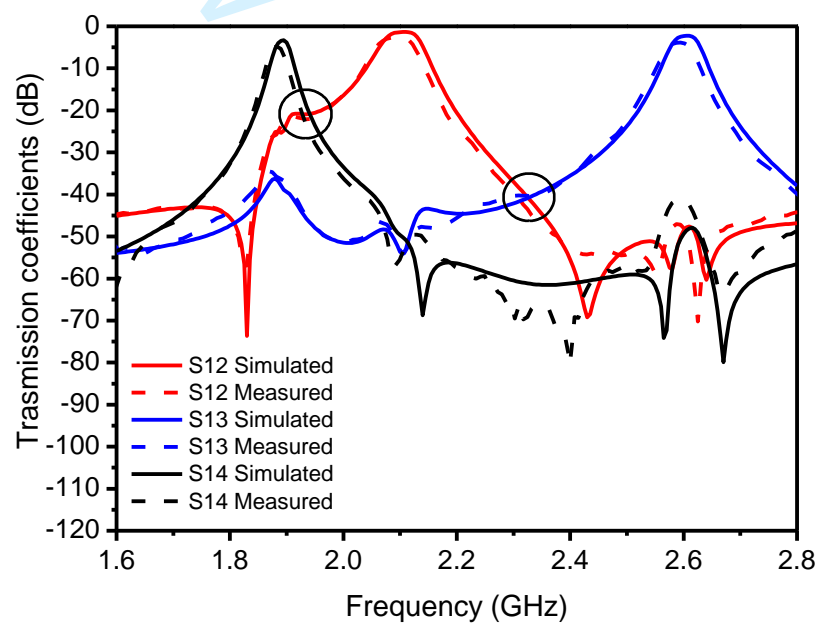

$a$ 

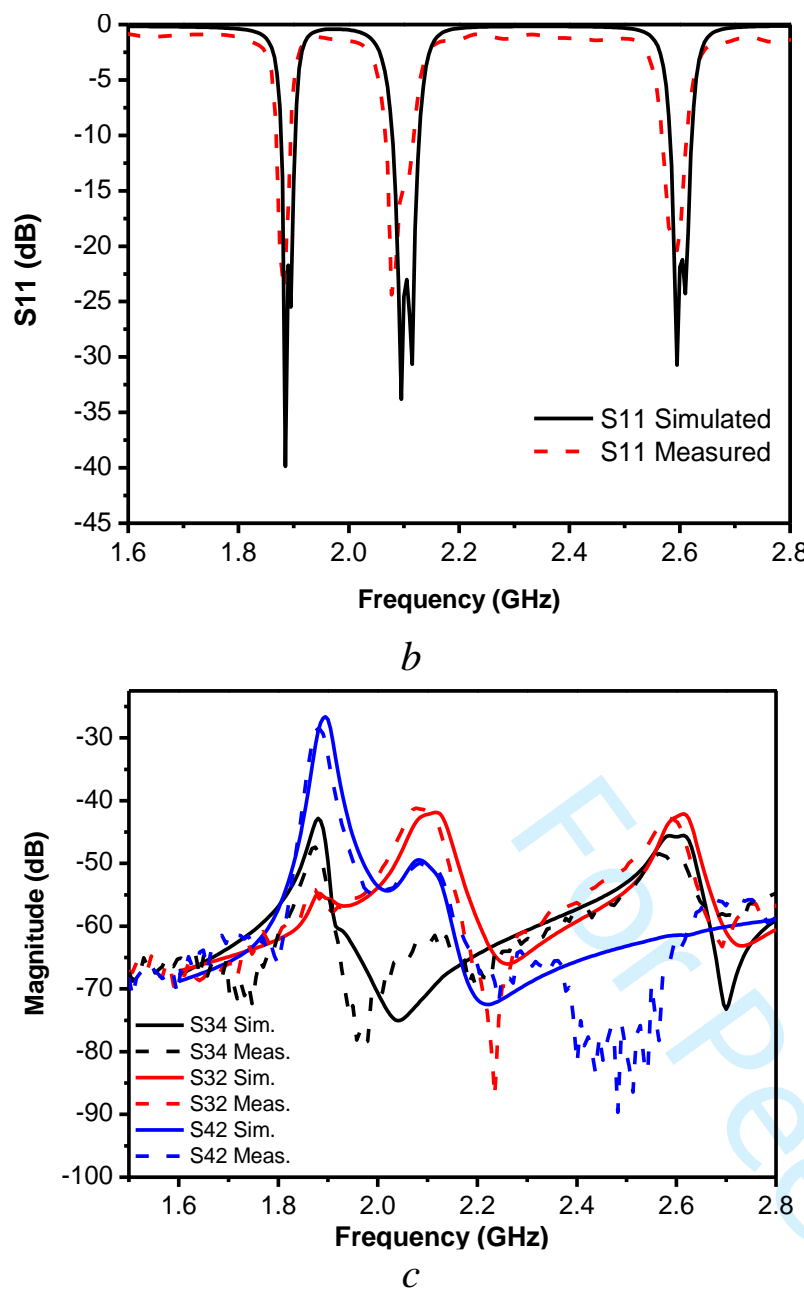

Fig.6.Triplexer measurement versus simulation.

(a) Transmission, (b) reflection, and (c) isolation between the three output ports

\section{Conclusion}

This letter proposes and analyses a double-stub-loaded resonator (DSLR) and uses it as the common resonator at a four-port triplexer. The analytical analysis and simulations have demonstrated the flexible control of the three resonances. Replacing the short stubs used in previous works with open stubs eases the implementation using microstrip technologies and still provides reasonably good isolation between the three bands. The simulated and measured results were found to be in good agreement.

\section{References}

[1] Cameron, R., Kudsia, C., and Mansour, R.: 'Microwave filters for communication systems' (Wiley, 2007)

[2] Wang, Y., Lancaster, M. J.: 'An Investigation on the coupling characteristics of a novel multiplexer configuration'. the 43rd European Microwave Conference, Nuremberg, Germany, Oct 2013, pp. 900903

[3] Shang, X., Wang, Y., Xia, W., et al.: 'Novel Multiplexer Topologies Based on All-Resonator Structures', IEEE Trans. Microwave Theo. Tech., 2013, 61, (11), pp. 3838-3845

[4] Skaik T., AbuHussain, M.: 'Design of diplexers for E-
Band Communication systems'. 13th Mediterranean Microwave Symposium (MMS), Saida, Lebanon, Sept. 2013, pp. 1-4

[5] Chuang, M. L., Wu, M. T.: 'Microstrip diplexer design using common T-shaped resonator', IEEE Microw. Wirel. Components Lett., 2011, 21, (11), pp. 583-585

[6] Kim, J. H., Kim, B. C., Byun, Y. T., et al.: 'Tri-band bandpass filter using stub loaded resonators', Electron. Lett., 2008, 43, (12), pp. 747-749

[7] Zhang, X. Y., Chen, J. X., Xue, Q., et al.: 'Dual-band bandpass filters using stub-loaded resonators', IEEE Microw. Wirel. Components Lett., 2007, 17, (8), pp. 583-585

[8] Mondal, P., Mandal, M. K.: 'Design of Dual-Band Bandpass Filters Using Stub-Loaded Open-Loop Resonators', IEEE Transactions on Microwave Theory and Techniques, 2008, 56, (1), pp. 150-155

[9] Gao, L., Zhang, X. Y., Xue, Q.: 'Compact Tri-Band Bandpass Filter Using Novel Eight-Mode Resonator for 5G WiFi Application', IEEE Microwave and Wireless Components Letters, 2015, 25, (10), pp. 660662

[10] Choocadee, S., Intarawiset, N., Tantiviwat, S.: 'Compact microstrip diplexer using triple-mode stub loaded resonators'. IEEE MTT-S International Conference on Microwaves for Intelligent Mobility, Nagoya, Japan, March 2017, pp. 9-12

[11] Lan, S. W., Weng, M.H., Chang, S. J., et al.: 'A tri-band bandpass filter with wide stopband using asymmetric stub-loaded resonators," IEEE Microw. Wirel. Components Lett., 2015, 25, (1), pp. 19-21

[12]Li, C., Chen, J., Tang, H., et al.: 'Tri-band bandpass fi lter with wide stop-band using stub-loaded triple-mode resonator', J. Electromagn. Waves Appl., 2013, 27, (4), pp. 439-447

[13] Doan, M., Nguyen, T., Le, H.: 'Miniaturized tri-band bandpass filter using modified triple-mode resonators with multiple transmission zeros'. International Conference on Advanced Technologies for Communications, Ho Chi Minh City, Vietnam, Oct. 2013, pp. 492-495

[14] Liu, W.Q., Wei, F., Shi, X. W.: 'A compact Tri-band power divider based on Triple-mode resonator', Prog. Electromagn. Res. C, 2013, 138, pp. 283-291

[15]Qian, J., Chen, F.: 'Wide Stopband Microstrip Triplexer Using Common Crossed Resonator and Uniform Impedance Resonator', Prog. Electromagn. Res. Lett.,2017, 69, pp. 79-86

[16]Zhang, X. Y., Chen, J. X., Xue, Q., et al.: 'Dual-Band Bandpass Filters Using Stub-Loaded Resonators', IEEE Microwave and Wireless Components Letters, 2007, 17, (8), pp. 583-585 